\title{
Effects of renin-angiotensin system blockers on the risk and outcomes of severe acute respiratory syndrome coronavirus 2 infection in patients with hypertension
}

Jinwoo Lee ${ }^{1,}$, Seong Jin Jo ${ }^{2,}$, Youngjin $\mathrm{Cho}^{3}$, Ji Hyun Lee ${ }^{3}$, Il-Young Oh³ , Jin Joo Park ${ }^{3}$, Young-Seok Cho ${ }^{3}$, and Dong-Ju Choi ${ }^{3}$

${ }^{1}$ Division of Pulmonary and Critical Care Medicine, Department of Internal Medicine, ${ }^{2}$ Department of Dermatology, Seoul National University College of Medicine, Seoul; ${ }^{3}$ Division of Cardiology, Department of Internal Medicine, Seoul National University Bundang Hospital, Seongnam, Korea

Received: July 31, 2020

Revised : August 17, 2020

Accepted: August 24, 2020

Correspondence to

Youngjin Cho, M.D.

Division of Cardiology,

Department of Internal

Medicine, Seoul National

University Bundang Hospital, 82

Gumi-ro 173beon-gil, Bundang-

gu, Seongnam 13620, Korea

Tel: +82-31-787-7083

Fax: +82-31-787-4290

E-mail: cho_y@snubh.org

https://orcid.org/0000-0001-

8106-3713

*These authors equally contributed to the study.
Background/Aims: There are concerns that the use of renin-angiotensin system (RAS) blockers may increase the risk of being infected with severe acute respiratory syndrome coronavirus 2 (SARS-CoV-2) or progressing to a severe clinical course after infection. This this study aimed to investigate the influence of RAS blockers on the risk and severity of SARS-CoV-2 infection.

Methods: We conducted a retrospective cohort study analyzing nationwide claims data of 215,184 adults who underwent SARS-CoV-2 tests in South Korea. The SARS-CoV-2 positive rates and clinical outcomes were evaluated according to the use of RAS blockers in patients with hypertension $(n=64,243)$.

Results: In total, 38,919 patients with hypertension were on RAS blockers. The SARS-CoV-2 positive rates were significantly higher in the RAS blocker group than in the control group after adjustments (adjusted odds ratio [OR], 1.22; 95\% confidence interval [CI], 1.10 to $1.36 ; p<0.001$ ), and matching by propensity score (adjusted OR, 1.16; 95\% CI, 1.03 to 1.32; $p=0.017$ ). Among the 1,609 SARS-CoV-2positive patients with hypertension, the use of RAS blockers was not associated with poor outcomes, such as mortality (adjusted OR, 0.81; 95\% CI, 0.56 to 1.17; $p=$ 0.265 ), and a composite of admission to the intensive care unit and mortality (adjusted OR, 0.95 ; $95 \% \mathrm{CI}, 0.73$ to $1.22 ; p=0.669$ ). Analysis in the propensity scorematched population showed consistent results.

Conclusions: In this Korean nationwide claims dataset, the use of RAS blockers was associated with a higher risk to SARS-CoV-2 infection but not with higher mortality or other severe clinical courses.

Keywords: Coronavirus infections; Hypertension; Angiotensin-converting enzyme inhibitor; Angiotensin receptor antagonists

\section{INTRODUCTION}

Severe acute respiratory syndrome coronavirus 2 (SARS$\mathrm{CoV}-2)$ infection has officially become a pandemic, with more than 2 million confirmed cases and more than 100,000 deaths worldwide. Elderly patients and those with underlying comorbidities such as cardiovascular disease and hypertension are considered to be at higher 
risk of morbidity and mortality from SARS-CoV-2 infection [1]. Many of these patients are on renin-angiotensin system (RAS) blockers, and the effect of angiotensin-converting enzyme (ACE) inhibitors and angiotensin receptor blockers (ARBs) on the clinical outcomes of patients with coronavirus disease (COVID-19) has been controversial. Given that the angiotensin-converting enzyme 2 (ACE2) is the cell entry receptor for SARS-CoV-2 virus [2]. there is growing concern that pre-existing use of ACE inhibitors and ARBs might increase the expression of ACE2 and, therefore, possibly increase the risk of being infected with SARS-CoV-2 and/or progressing to a severe and fatal course after infection [3-5].

As a response to this concern, there have been recommendations for both the continued use and cessation of ACE inhibitors and ARBs among patients with COVID-19 [4,6]. Unfortunately, current evidence is insufficient to either support or oppose this concern. To date, there are only a few human studies evaluating the effect of RAS blockers on patients with COVID-19. Several studies have shown that patients on RAS blockers did not have a higher risk of severe COVID-19 than those who did not receive any RAS blockers [7-10]. However, whether RAS blockers increases the risk of being infected with SARS-CoV-2 infection is challenging to answer. Therefore, this study aimed to determine the influence of RAS blockers on the risk and severity of SARS-CoV-2 infection, using medical claims data.

\section{METHODS}

\section{Data source and study population}

The South Korean healthcare system is governed by the Ministry of Health and Welfare and covers approximately $97 \%$ of the overall Korean population. The claims data of Health Insurance Review and Assessment Service (HIRA) contains comprehensive information such as patients' diagnosis, procedures, surgeries, treatment, and prescription history. Recently, the Ministry of Health and Welfare and HIRA initiated a global research collaboration "OpenData4Covidı" project (https://hira-covid19.net/). This project provides, via their website, insurance claims data for those who tested for SARSCoV-2 infection, along with all the claim records up to 3 years before the individual's date of SARS-CoV-2 test.
As of May 27, 2020, data up to May 15, 2020 was released, and Korea Centers for Disease Control \& Prevention (KCDC) data was merged to HIRA claims data with respect to SARS-CoV-2 confirmation and mortality.

The dataset included data of 234,427 subjects who had been tested for SARS-CoV-2 infection. Among 215,184 patients older than 19 years, 64,243 patients with hypertension were included in the study. All SARS-CoV-2 infection cases were confirmed via reverse transcriptase polymerase chain reaction. This study was approved by the Institutional Review Board of Seoul National University Bundang Hospital (IRB No. X-2005-613-902) and conformed to the tenets of the Declaration of Helsinki. The requirement for informed consent was waived off as all study participants were anonymized.

\section{Definitions of SARS-CoV-2 cases, comorbidities, and clinical outcomes}

SARS-CoV-2 positive cases were predefined in the provided dataset, based on both COVID-19 case data from the KCDC. Claims data for the prescription of ACE inhibitors or ARBs were screened. Subjects were confirmed to be on ACE inhibitors or ARBs if their prescription had covered more than 4 weeks of an 8-week duration before the SARS-CoV-2 test (medication possession ratio $>50 \%$ ). Prescription data on other classes of antihypertensive medication (beta-blocker, calcium-channel blocker, and diuretics) were also screened in the same manner.

Baseline comorbidities were also defined using ICD10 codes as follows: hypertension, I10-13, I15; heart failure, I50; history of myocardial infarction, I21, 22; and diabetes mellitus, E11-14. Chronic obstructive pulmonary disease (COPD) and asthma were defined using a combination of ICD-10 codes (J43.1-9, J44 for COPD; J45, J46 for asthma) and prescription history of relevant drugs at least twice in 2019. The detailed claim codes used for the definitions are provided as a supplementary file.

This study has two main outcomes: [1] positive SARSCoV-2 test among subjects that had been tested, and [2] mortality among SARS-CoV-2 (+) patients. The secondary outcome measure was severe clinical course among SARS-CoV-2 (+) patients, defined as a composite of requiring oxygen therapy, admission to the intensive care unit(ICU), and mortality. A composite of ICU admission and mortality were also evaluated. The flowchart of the 

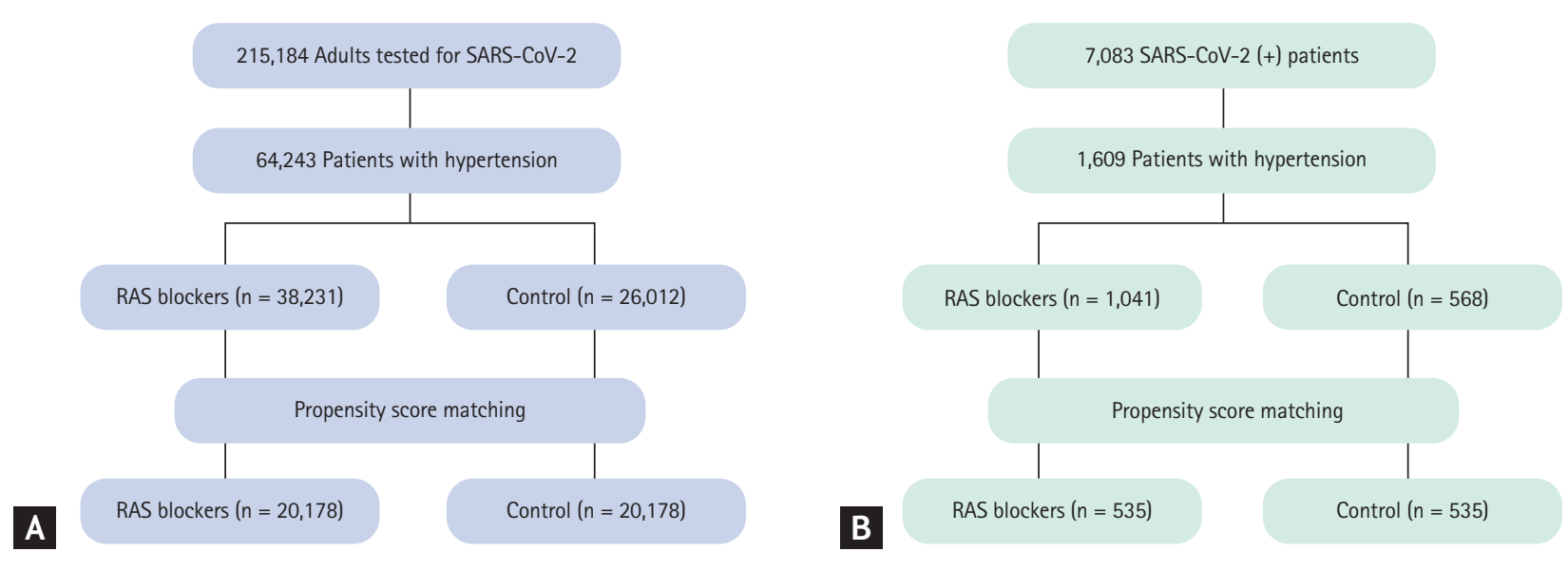

Figure 1. Schematic figure of the study population. (A) The risk of renin-angiotensin system blockers for severe acute respiratory syndrome coronavirus 2 (SARS-CoV-2) infection was evaluated in patients with hypertension who had been tested for SARS-CoV-2. (B) The risk for severe clinical courses were evaluated in SARS-CoV-2 (+) patients. RAS, renin-angiotensin system.

scheme of the study is presented in Fig. 1.

\section{Statistical analysis}

Pearson's chi-square test or Fisher's exact test were used for categorical variables, and the Student's $t$ test was used for continuous variables. Outcomes from logistic regression were presented as odds ratios (ORs) with 95\% confidence intervals (CIs). Comparison between RAS blocker user and non-user were performed in patients with hypertension. To address potential sources of bias, propensity score matching was additionally performed. The baseline clinical characteristics were incorporated into a non-parsimonious logistic regression model to compute the propensity score for the use of RAS blocker. The included covariates were age, sex, diabetes mellitus, heart failure, history of myocardial infarction, asthma or COPD, and use of other classes of antihypertensive medications. Then, propensity score-matching iteration from the eighth digit to the third digit in the SARS-CoV-2 tested population, and to the first digit in the SARS-CoV-2 (+) population were performed. In total, 20,178 matched pairs regarding the use of RAS blocker were selected from the overall SARS-CoV-2 tested population (c-statistics $=0.671$ ) and 535 pairs from those that tested positive for SARS-CoV-2 (c-statistics $=0.666)$. The baseline characteristics and outcomes were compared again between the patients who were not on RAS blockers (control group) and the patients on RAS blockers (RAS blocker group) at the time of SARS-CoV-2 testing.
All statistical tests were performed using SAS version 9.4 (SAS Institute, Cary, NC, USA), and $p<0.05$ was considered statistically significant.

\section{RESULTS}

\section{Baseline characteristics}

In total, 38,231 (59.5\%) patients with hypertension were on ACE inhibitors or ARBs at the time of testing. The comparison of baseline characteristics between the RAS blocker user and non-user in the overall hypertensive and matched population are presented in Table 1. In the overall hypertensive population, the RAS blocker group patients were younger than those of the control group. Male sex and diabetes mellitus were more prevalent, and the proportion of heart failure and COPD/asthma were lower in the RAS blocker group. Most patients (96.3\%) in the RAS blocker group used ARBs. After matching by propensity score, these parameters became comparable between the groups.

\section{Risk of SARS-CoV-2 infection}

The ORs for positive SARS-CoV-2 tests are presented in Table 2. The positive rate of SARS-CoV-2 tests was significantly higher in the RAS blockers group than in the control group before (OR, 1.25; 95\% CI, 1.13 to 1.39; $p<$ 0.001) and after adjustment for age, sex, diabetes, heart failure, history of myocardial infarction, airway diseases 
Table 1. The comparison of baseline characteristics between RAS blocker user and non-user in the overall hypertensive and matched population

\begin{tabular}{|c|c|c|c|c|c|c|}
\hline \multirow[b]{2}{*}{ Characteristic } & \multicolumn{3}{|c|}{ Overall patients with hypertension } & \multicolumn{3}{|c|}{ Matched population } \\
\hline & $\begin{array}{l}\text { RAS blocker } \\
(\mathrm{n}=38,231)\end{array}$ & $\begin{array}{c}\text { Control } \\
(\mathrm{n}=26,012)\end{array}$ & $p$ value & $\begin{array}{l}\text { RAS blocker } \\
(\mathrm{n}=20,178)\end{array}$ & $\begin{array}{c}\text { Control } \\
(\mathrm{n}=20,178)\end{array}$ & $p$ value \\
\hline Age, yr & $66.8 \pm 14.7$ & $69.3 \pm 14.9$ & $<0.001$ & $68.9 \pm 13.7$ & $68.9 \pm 13.7$ & 0.783 \\
\hline Male sex & $20,067(52.5)$ & $13,431(51.6)$ & 0.033 & $10,205(50.6)$ & $10,230(50.7)$ & 0.803 \\
\hline Heart failure & $5,315(13.9)$ & $4,054(15.6)$ & $<0.001$ & 2,397 (11.9) & $2,419(12.0)$ & 0.736 \\
\hline Prior MI & $2,377(6.2)$ & $1,689(6.5)$ & 0.159 & $858(4 \cdot 3)$ & $842(4.2)$ & 0.692 \\
\hline Diabetes & $17,731(46.4)$ & $11,000(42.3)$ & $<0.001$ & $8,729(43 \cdot 3)$ & $8,720(43 \cdot 2)$ & 0.928 \\
\hline COPD or asthma & $7,028(18.4)$ & $5,181(19 \cdot 9)$ & $<0.001$ & $3,530(17.5)$ & $3,527(17.5)$ & 0.969 \\
\hline RAS blocker & $38,231(100)$ & O & $<0.001$ & $20,178(100)$ & o & $<0.001$ \\
\hline ACEi & $1,626(4 \cdot 3)$ & o & $<0.001$ & $935(4.6)$ & o & $<0.001$ \\
\hline ARB & $36,811(96.3)$ & $\mathrm{O}$ & $<0.001$ & $19,316(95.7)$ & o & $<0.001$ \\
\hline Beta-blocker & $9,366(24 \cdot 5)$ & $6,017(23.1)$ & $<0.001$ & $4,224(20.9)$ & $4,233(21.0)$ & 0.912 \\
\hline Calcium-channel blocker & $22,556(59.0)$ & $11,662(44.8)$ & $<0.001$ & $10,379(51.4)$ & $10,370(51.4)$ & 0.929 \\
\hline Diuretics & $8,566(22.4)$ & $1,250(4.8)$ & $<0.001$ & $1,032(5.1)$ & $1,032(5.1)$ & 1.000 \\
\hline SARS-CoV-2 (+) & $1,041(2.7)$ & $568(2.2)$ & $<0.001$ & $547(2.7)$ & $472(2.3)$ & 0.017 \\
\hline
\end{tabular}

Values are presented as mean $\pm \mathrm{SD}$ or number $(\%)$.

RAS, renin-angiotensin system; MI, myocardial infarction; COPD, chronic obstructive pulmonary disease; ACEi, angiotensin-converting enzyme inhibitor; ARB, angiotensin receptor blocker; SARS-CoV2, severe acute respiratory syndrome coronavirus 2.

(COPD or asthma), and other classes of antihypertensive medications (OR, 1.22; 95\% CI, 1.10 to $1.36 ; p<0.001$ ). Other than RAS blocker usage, younger age and female sex were associated with higher SARS-CoV-2 positivity rates in the multivariate analysis. Diabetes mellitus, cardiovascular and airway diseases were correlated with lower SARS-CoV-2 positive tests. Analysis in the propensity score-matched population also showed significantly higher SARS-CoV-2 positive rate in the RAS blocker group than that in the control group (OR, 1.16; 95\% CI, 1.03 to $1.32 ; p=0.017$ ).

\section{Clinical outcomes in the SARS-CoV-2-positive pop- ulation}

In total, 7,083 subjects tested positive for SARS-CoV-2 infection, and among them, 1,609 patients had history of hypertension. The clinical outcomes were compared according to the use of RAS blockers in the SARS-CoV-2 (+) patients with hypertension. The baseline characteristics of the overall SARS-CoV-2 (+) with hypertension and the matched population are presented in Table 3.

The overall mortality rate in this dataset was 3.2\% $(227 / 7,083)$. Among patients with hypertension, the mor- tality rate was $10.3 \%(165 / 1,609)$, and the predictors for mortality are shown in Table 4. Use of beta-blocker, older age, diabetes mellitus, cardiovascular disease, and airway diseases were significantly associated with a higher mortality in the univariate logistic regression analysis. In multivariate analysis, age (per 10 years, adjusted OR, 3.11; 95\% CI, 2.56 to 3.79; $p<0.001$ ), diabetes mellitus (adjusted OR, 2.24; 95\% CI, 1.56 to 3.22; $p<0.011$ ), and airway diseases (adjusted OR, 1.95; 95\% CI, 1.21 to 3.13; $p=0.006$ ) were significantly associated with higher mortality. Patients on RAS blockers showed lower mortality in the univariate analysis (OR, 0.59; 95\% CI, 0.43 to $0.82 ; p=$ $0.001)$, but there was no significant difference after adjustments for comorbidities and other classes of medications (adjusted OR, $0.81 ; 95 \%$ CI, 0.56 to 1.17; $p=0.265$ ) or matching by propensity score (OR, o.88; $95 \%$ CI, 0.59 to $1.33 ; p=0.545$ ). With respect to a composite of ICU admission and mortality, there was no significant difference between the RAS blocker and the control groups after adjustment or propensity score matching (adjusted OR, $0.95 ; 95 \%$ CI, 0.73 to 1.22; $p=0.669$ ) (adjusted OR, 1.05; 95\% CI, 0.79 to 1.40; $p=0.720$ ). Analyses on severe clinical course (a composite of oxygen therapy, ICU ad- 
Table 2. Clinical factors associated with SARS-CoV-2 infection in the overall and matched population

\begin{tabular}{|c|c|c|c|c|c|c|}
\hline \multirow[b]{2}{*}{ Variable } & \multicolumn{4}{|c|}{ Overall patients with hypertension $(n=64,243)$} & \multicolumn{2}{|c|}{ Matched population $(n=40,356)$} \\
\hline & $\begin{array}{l}\text { Unadjusted OR } \\
(95 \% \mathrm{CI})\end{array}$ & $p$ value & $\begin{array}{c}\text { Adjusted OR } \\
(95 \% \mathrm{CI})\end{array}$ & $p$ value & $\begin{array}{c}\text { Adjusted OR } \\
(95 \% \mathrm{CI})\end{array}$ & $p$ value \\
\hline RAS blocker & $1.25(1.13-1.39)$ & $<0.001$ & $1.22(1.10-1.36)$ & $<0.001$ & $1.16(1.03-1.32)$ & 0.017 \\
\hline Age, /10 yr & $0.92(0.89-0.95)$ & $<0.001$ & $0.95(0.91-0.98)$ & 0.001 & $0.96(0.92-1.01)$ & 0.089 \\
\hline Female sex & $1.43(1.30-1.58)$ & $<0.001$ & $1.44(1.30-1.59)$ & $<0.001$ & $1.35(1.19-1.54)$ & $<0.001$ \\
\hline HF or prior MI & $0.57(0.49-0.66)$ & $<0.001$ & $0.68(0.57-0.80)$ & $<0.001$ & $0.66(0.53-0.82)$ & $<0.001$ \\
\hline Diabetes & $0.80(0.72-0.88)$ & $<0.001$ & $0.86(0.78-0.96)$ & 0.005 & $0.84(0.73-0.95)$ & 0.008 \\
\hline COPD or asthma & $0.47(0.40-0.56)$ & $<0.001$ & $0.52(0.44-0.61)$ & $<0.001$ & $0.49(0.39-0.61)$ & $<0.001$ \\
\hline Beta-blocker & $0.76(0.67-0.86)$ & $<0.001$ & $0.89(0.78-1.01)$ & 0.069 & $0.96(0.81-1.14)$ & 0.608 \\
\hline Calcium-channel blocker & $0.99(0.89-1.09)$ & 0.761 & $0.95(0.86-1.05)$ & 0.288 & $1.11(0.98-1.26)$ & 0.103 \\
\hline Diuretics & $1.21(1.06-1.38)$ & 0.004 & $1.12(0.98-1.28)$ & 0.106 & $1.01(0.76-1.34)$ & 0.935 \\
\hline
\end{tabular}

SARS-CoV-2, severe acute respiratory syndrome coronavirus 2; OR, odds ratio; CI, confidence interval; RAS, renin-angiotensin system; HF, heart failure; MI, myocardial infarction; COPD, chronic obstructive pulmonary disease.

Table 3. Baseline characteristics of SARS-CoV-2 positive population

\begin{tabular}{|c|c|c|c|c|c|c|}
\hline \multirow[b]{2}{*}{ Variable } & \multicolumn{3}{|c|}{ SARS-CoV-2 (+) patients with hypertension } & \multicolumn{3}{|c|}{ Matched population } \\
\hline & $\begin{array}{l}\text { RAS blocker } \\
(\mathrm{n}=1,041)\end{array}$ & $\begin{array}{l}\text { Control } \\
(\mathrm{n}=568)\end{array}$ & $p$ value & $\begin{array}{l}\text { RAS blocker } \\
(\mathrm{n}=535)\end{array}$ & $\begin{array}{l}\text { Control } \\
(\mathrm{n}=535)\end{array}$ & $p$ value \\
\hline Age, yr & $64.6 \pm 12.7$ & $69.0 \pm 14.1$ & $<0.001$ & $68.3 \pm 12.4$ & $68.2 \pm 13.9$ & 0.871 \\
\hline Male sex & $455(43.7)$ & $244(43.0)$ & 0.772 & $239(44 \cdot 7)$ & $235(43.9)$ & 0.806 \\
\hline Heart failure & $84(8.1)$ & $55(9.7)$ & 0.271 & $51(9.5)$ & $52(9 \cdot 7)$ & 0.917 \\
\hline Prior MI & $39(3.8)$ & $26(4 \cdot 6)$ & 0.418 & $24(4 \cdot 5)$ & $22(4 \cdot 1)$ & 0.763 \\
\hline Diabetes & $420(40.4)$ & $212(37 \cdot 3)$ & 0.236 & $210(39.2)$ & $201(37.6)$ & 0.572 \\
\hline COPD or asthma & $105(10.1)$ & $5^{8}(10.2)$ & 0.937 & $53(9.9)$ & $56(10.5)$ & 0.762 \\
\hline RAS blocker & $1,041(17.8)$ & o & $<0.001$ & $535(100)$ & o & $<0.001$ \\
\hline ACEi & $45(4 \cdot 3)$ & 0 & $<0.001$ & $35(6.5)$ & 0 & $<0.001$ \\
\hline ARB & $998(95 \cdot 9)$ & o & $<0.001$ & $502(93.8)$ & o & $<0.001$ \\
\hline Beta-blocker & $185(17.8)$ & $127(40.7)$ & 0.026 & $117(21.9)$ & $110(20.6)$ & 0.601 \\
\hline Calcium-channel blocker & $556(53 \cdot 4)$ & $295(51.9)$ & 0.572 & $280(52.3)$ & $285(53 \cdot 3)$ & 0.760 \\
\hline Diuretics & $250(24.0)$ & $37(6.5)$ & $<0.001$ & $39(7 \cdot 3)$ & $37(6.9)$ & 0.812 \\
\hline
\end{tabular}

Values are presented as mean $\pm \mathrm{SD}$ or number $(\%)$.

SARS-CoV-2, severe acute respiratory syndrome coronavirus 2; RAS, renin-angiotensin system; MI, myocardial infarction; COPD, chronic obstructive pulmonary disease; ACEi, angiotensin-converting enzyme inhibitor; ARB, angiotensin receptor blocker.

mission, and mortality) showed similar trends (Fig. 2).

\section{Analysis on the effects of other classes of antihyper- tensive medications}

Baseline characteristics after matching by propensity score for each class of antihypertensive medications in the SARS-CoV-2 tested and the SARS-CoV-2 (+) popula- tion with hypertension are provided as Supplementary Tables 1 and 2. Other classes of antihypertensive medications were not associated with the rate of SARS-CoV-2 positive tests (Supplementary Table 3). Clinical outcomes of SARS-CoV-2 infection were also not associated with the use of other classes of antihypertensive medications after adjustments for comorbidities and other 
Table 4. Predictors of death in SARS-CoV-2 (+) patients with hypertension

\begin{tabular}{|c|c|c|c|c|}
\hline \multirow{2}{*}{ Variable } & \multicolumn{4}{|c|}{ SARS-CoV-2 (+) with hypertension $(\mathrm{n}=1,609)$} \\
\hline & Unadjusted OR (95\% CI) & $p$ value & Adjusted OR (95\% CI) & $p$ value \\
\hline RAS blocker & $0.59(0.43-0.82)$ & 0.001 & $0.81(0.56-1.17)$ & 0.265 \\
\hline Age, /10 yr & $2.87(2.41-3.41)$ & $<0.001$ & $3.11(2.56-3.79)$ & $<0.001$ \\
\hline Female sex & $0.76(0.55-1.04)$ & 0.088 & $0.47(0.32-0.68)$ & $<0.001$ \\
\hline HF or prior MI & $2.64(1.77-3.94)$ & $<0.001$ & $1.34(0.82-2.19)$ & 0.244 \\
\hline Diabetes & $2.23(1.61-3.09)$ & $<0.001$ & $2.24(1.56-3.22)$ & $<0.001$ \\
\hline COPD or asthma & $2.65(1.74-4.02)$ & $<0.001$ & $1.95(1.21-3.13)$ & 0.006 \\
\hline Beta-blocker & $1.49(1.02-2.16)$ & 0.039 & $1.21(0.78-1.89)$ & 0.404 \\
\hline Calcium-channel blocker & $0.87(0.63-1.20)$ & 0.386 & $0.87(0.60-1.24)$ & 0.437 \\
\hline Diuretics & $1.03(0.68-1.56)$ & 0.902 & $1.12(0.70-1.80)$ & 0.637 \\
\hline
\end{tabular}

SARS-CoV-2, severe acute respiratory syndrome coronavirus 2; OR, odds ratio; CI, confidence interval; RAS, renin-angiotensin system; HF, heart failure; MI, myocardial infarction; COPD, chronic obstructive pulmonary disease.

\begin{tabular}{|c|c|c|c|c|}
\hline & & $\begin{array}{l}\text { RAS blocker } \\
\text { (no./total no.) }\end{array}$ & $\begin{array}{c}\text { Control } \\
\text { (no./total no.) }\end{array}$ & $\begin{array}{c}\text { Adjusted OR } \\
(95 \% \mathrm{CI})\end{array}$ \\
\hline \multirow[t]{2}{*}{ Infection risk } & HTN & $1,041 / 38,231$ & $568 / 26,012$ & $1.22(1.10-1.36)$ \\
\hline & Matched & $547 / 20,178$ & $472 / 20,178$ & $1.16(1.03-1.32)$ \\
\hline \multirow[t]{2}{*}{ Death } & HTN & $88 / 1,041$ & $77 / 568$ & $0.81(0.56-1.17)$ \\
\hline & Matched & $61 / 535$ & $66 / 535$ & $0.88(0.59-1.33)$ \\
\hline \multirow[t]{2}{*}{ ICU/Death } & HTN & $228 / 1,041$ & $150 / 568$ & $0.95(0.73-1.22)$ \\
\hline & Matched & $141 / 535$ & $135 / 535$ & $1.05(0.79-1.40)$ \\
\hline \multirow[t]{2}{*}{$\mathrm{O}_{2} / \mathrm{ICU} /$ Death } & HTN & $378 / 1,041$ & $236 / 568$ & $1.01(0.80-1.27)$ \\
\hline & Matched & $219 / 535$ & $220 / 535$ & $0.99(0.77-1.28)$ \\
\hline
\end{tabular}

Figure 2. Risk of renin-angiotensin system (RAS) blockers for severe acute respiratory syndrome coronavirus 2 (SARS-CoV-2) infection and its outcomes. The use of RAS blockers was associated with higher SARS-CoV-2 infection rate but was not associated with higher mortality or other severe clinical courses in both patients with hypertension and propensity score matched population. OR, odds ratio; CI, confidence interval; HTN, hypertension; ICU, intensive care unit.

classes of medications or matching by propensity score (Supplementary Table 4).

\section{DISCUSSION}

Hypertension is one of the most prevalent comorbid ities in COVID-19 patients and is also associated with poor outcomes [1]. Given that the ACE2 is the cell entry receptor for SARS-CoV-2 virus and that ACE inhibitors and ARBs are frequently used to manage hypertension, the use of RAS blockers in patients with COVID-19 has been controversial. Recent studies suggested that the use of RAS blockers was not associated with poor outcomes in hospitalized COVID-19 patients [7,8]. However, clinical evidence is still insufficient to determine if there is an association between the use of RAS blockers and COVID-19. Moreover, the effect of RAS blockers on the risk of being infected with SARS-CoV-2 is more difficult to determine from lack of data. In the current study, we analyzed HIRA claims data of those tested for SARS-CoV-2 infection and found that the use of RAS blockers may increase the risk of SARS-CoV-2 infection. However, it is not associated with more severe clinical courses. These results support the theory that there may be a dual role of ACE inhibitors/ARBs in COVID-19 [11]. 


\section{RAS blockers and the risk of SARS-CoV-2 infection}

ACE2 is the functional receptor for SARS-CoV-2 cell entry [2], and although the results are inconsistent, select preclinical animal studies showed higher ACE2 expression after the use of RAS blockers [12-14]. In contrast, data from human studies showed that RAS blockers have little to no effect on urinary or plasma ACE2 levels [15]. It is also uncertain if changes in urinary or plasma ACE2 levels reflect changes in tissue ACE2. Despite insufficient evidence, there are still concerns about the use of RAS blockers, particularly that they may alter ACE2 and play a role in COVID-19. In this study, the use of RAS blockers was associated with a significantly higher rate of SARS-CoV-2 infection after adjustment or matching by propensity score, indicating that more caution may be needed while managing patients on RAS blockers.

In contrast, a recent studies from Italy and US reported that the use of RAS blockers did not affect the risk of developing COVID-19 [9,16]. The systematic review from these studies also showed similar outcomes [17]. The disparity in results may be explained by the differences in the study population. The Korean government has conducted extensive testing and tracing measures, including the use of advanced information technology [18] to control the spread of the virus. This enabled the identification of a large group of individuals who are suspected to have been exposed to, but not infected with, SARS-CoV-2 as the control group. In contrast, in the previously mentioned Italian study [16], the subjects not tested for SARS-CoV-2 infection were designated as the control group and those infected with SARS-CoV-2 may have been unintentionally included. The Italian Ministry of Health had issued stringent testing policies, and testing was limited to those with mild symptoms or did not require hospitalization [19]. Tests for SARSCoV-2 were also limited in the US and contact tracing was not widely available [20]. In the study by Reynolds et al. [9], $46.8 \%$ of the patients tested for COVID-19 were positive. In contrast, the positive rate was $3.3 \%$ in our study population, suggesting that this dataset is well designed to assess the risk of SARS-CoV-2 infection. It was suggested in the systematic review that previous studies were mostly from symptomatic patients and therefore these results may not apply to patients with mild or no symptoms [17].

Another study from Korea reported that use of RAS blockers was not associated with the increased risk of COVID-19 [21]. The previous study included individuals without hypertension and those that were not tested for SARS-CoV-2 infection. Moreover, the underlying comorbidities of the population were not assessed and there were no adjustments for comorbidities or other antihypertensive medications.

Other than the use of RAS blockers, age, sex, diabetes, cardiovascular and airway diseases were also associated with higher risk of SARS-CoV-2 infection. Comorbidities suggested as risk factors for severe COVID-19 were associated with lower SARS-CoV-2 infection rate in our population. There is no clear biological explanation for this. One possible explanation is that changes in behavioral pattern may have contributed to these results. Subjects at higher risk for severe outcomes might had been more cautious than healthy subjects with respect to personal protection and hygiene during this pandemic. A recent report on the association between risk perception of COVID-19 and self-protective behaviors may support this explanation [22].

\section{RAS blockers and clinical outcomes}

In addition to the risk of infection, the use of RAS blockers was also suggested to affect the clinical course of SARS-CoV-2 infection [4,5], and some media sources even recommended discontinuation of RAS blockers in patients with known or suspected COVID-19. Despite these theoretical concerns and uncertainties, there is evidence of potential benefit, rather than harm, of RAS blockers in the treatment of COVID-19. In preclinical studies, RAS blockade attenuated SARS-CoV-1-induced acute lung injury [23] and other viral-induced lung injuries [24]. Previous studies have suggested that the use of RAS blockers did not have an adverse effect on the clinical course of COVID-19 [8-10,16]. There were even suggestions that RAS blockers were associated with a lower mortality rate [7]. This study also showed that the pre-existing use of RAS blockers did not increase the risk of mortality, admission to the ICU, or oxygen therapy. These findings support the recommendations from relevant professional societies that advocated for the continued use of ACE inhibitors/ARBs unless clinically indicated [25].

\section{Characteristics of the study population}

It is noteworthy that the general trend of SARS-CoV-2 
data in South Korea is different from those in China, Italy, Spain, or United States. The proportion of positive test results and case fatality rate were relatively lower, and the overall population was younger [19]. South Korea had experienced an exponential spread of SARSCoV-2 infection earlier than most countries. By late February 2020, the number of COVID-19 infections was the second highest after China. Using the extensive testing and tracing measures, the spread of virus was controlled without overwhelming the capacity of the healthcare system, which may have translated into the relatively lower fatality rate than that in other countries. Therefore, our data may represent the effects of the RAS blockers on the clinical course of COVID-19 in the setting of adequate healthcare management.

\section{Limitations}

This study had some limitations. This is an observational study, thus there may be additional unknown confounders or bias affecting our results despite propensity score matching. Since most of the study population was Asian, it is not known if the results could be applied to population of other ethnicities. Also, the effects of RAS blockers on SARS-CoV-2 infection may be different across the strains of COVID-19. The risk of infection may be influenced by the preventive measures taken by each individual. As this study was based on claims data, additional beneficiaries' information such as severity of conditions, laboratory results, and smoking status was not available. In addition, the use of RAS blockers was determined by prescription and may not coincide with the actual drug use. Dosage or adherence to drugs were not evaluated in this study. Further, less than $5 \%$ of subjects in the RAS blocker group were prescribed ACE inhibitors, and thus it was not feasible to assess the effect of ACE inhibitors and ARBs separately.

\section{Conclusions}

In this Korean nationwide claims dataset, the use of RAS blockers was associated with a higher risk of SARSCoV-2 infection but not with higher mortality or other severe clinical courses. Possible precautions should be taken in patients on RAS blockers, in order to reduce their exposure to SARS-CoV-2, and help control the spread of COVID-19.

\section{KEY MESSAGE}

1. The use of renin-angiotensin system (RAS) blockers was associated with a higher risk of severe acute respiratory syndrome coronavirus 2 (SARS-CoV-2) infection. However, the use of RAS blockers was not a risk factor for severe clinical courses.

2. These findings support the recommendations for continued use of RAS blockers in patients with hypertension unless clinically indicated. Nevertheless, possible precautions should be taken in patients on RAS blockers, in order to reduce their exposure to SARS-CoV-2 and help control the spread of coronavirus disease.

\section{Conflict of interest}

No potential conflict of interest relevant to this article was reported.

\section{Acknowledgments}

The authors thank the Ministry of Health and Welfare and the Health Insurance Review \& Assessment Service of Korea for sharing invaluable national health insurance claims data. We also thank the healthcare professionals dedicated to treating COVID-19 patients in Korea.

\section{REFERENCES}

1. Yang J, Zheng Y, Gou X, et al. Prevalence of comorbidities and its effects in patients infected with SARS-CoV-2: a systematic review and meta-analysis. Int J Infect Dis 2020;94:91-95.

2. Hoffmann M, Kleine-Weber H, Schroeder S, et al. SARSCoV-2 cell entry depends on ACE2 and TMPRSS2 and is blocked by a clinically proven protease inhibitor. Cell 2020;181:271-280.

3. Zheng YY, Ma YT, Zhang JY, Xie X. COVID-19 and the cardiovascular system. Nat Rev Cardiol 2020;17:259-260.

4. Fang L, Karakiulakis G, Roth M. Are patients with hypertension and diabetes mellitus at increased risk for COVID-19 infection? Lancet Respir Med 2020;8:e21.

5. Diaz JH. Hypothesis: angiotensin-converting enzyme inhibitors and angiotensin receptor blockers may increase 
the risk of severe COVID-19. J Travel Med 2020;27:taaao41.

6. Patel AB, Verma A. COVID-19 and angiotensin-converting enzyme inhibitors and angiotensin receptor blockers: what is the evidence? JAMA 2020;323:1769-1770.

7. Zhang P, Zhu L, Cai J, et al. Association of inpatient use of angiotensin-converting enzyme inhibitors and angiotensin II receptor blockers with mortality among patients with hypertension hospitalized with COVID-19. Circ Res 2020;126:1671-1681.

8. Li J, Wang X, Chen J, Zhang H, Deng A. Association of renin-angiotensin system inhibitors with severity or risk of death in patients with hypertension hospitalized for coronavirus disease 2019 (COVID-19) infection in Wuhan, China. JAMA Cardiol 2020;5:1-6.

9. Reynolds HR, Adhikari S, Pulgarin C, et al. Renin-angiotensin-aldosterone system inhibitors and risk of Covid-19. N Engl J Med 2020;382:2441-2448.

10. de Abajo FJ, Rodriguez-Martin S, Lerma V, et al. Use of renin-angiotensin-aldosterone system inhibitors and risk of COVID-19 requiring admission to hospital: a case-population study. Lancet 2020;395:1705-1714.

11. Guo J, Huang Z, Lin L, Lv J. Coronavirus disease 2019 (COVID-19) and cardiovascular disease: a viewpoint on the potential influence of angiotensin-converting enzyme inhibitors/angiotensin receptor blockers on onset and severity of severe acute respiratory syndrome coronavirus 2 infection. J Am Heart Assoc 2020;9:e016219.

12. Ferrario CM, Jessup J, Gallagher PE, et al. Effects of renin-angiotensin system blockade on renal angiotensin-(1-7) forming enzymes and receptors. Kidney Int 2005;68:2189-2196.

13. Ocaranza MP, Godoy I, Jalil JE, et al. Enalapril attenuates downregulation of angiotensin-converting enzyme 2 in the late phase of ventricular dysfunction in myocardial infarcted rat. Hypertension 2006;48:572-578.

14. Han SX, He GM, Wang T, et al. Losartan attenuates chronic cigarette smoke exposure-induced pulmonary arterial hypertension in rats: possible involvement of an- giotensin-converting enzyme-2. Toxicol Appl Pharmacol 2010;245:100-107.

15. Sriram K, Insel PA. Risks of ACE inhibitor and ARB usage in COVID-19: evaluating the evidence. Clin Pharmacol Ther 2020;108:236-241.

16. Mancia G, Rea F, Ludergnani M, Apolone G, Corrao G. Renin-angiotensin-aldosterone system blockers and the risk of Covid-19. N Engl J Med 2020;382:2431-2440.

17. Mackey K, King VJ, Gurley S, et al. Risks and impact of angiotensin-converting enzyme inhibitors or angiotensin-receptor blockers on SARS-CoV-2 infection in adults: a living systematic review. Ann Intern Med 2020;173:195203.

18. Park S, Choi GJ, Ko H. Information technology-based tracing strategy in response to COVID-19 in South Korea: privacy controversies. JAMA 2020;323:2129-2130.

19. Onder G, Rezza G, Brusaferro S. Case-fatality rate and characteristics of patients dying in relation to COVID-19 in Italy. JAMA 2020;323:1775-1776.

20. Dyer O. Covid-19: US testing ramps up as early response draws harsh criticism. BMJ 2020;368:m1167.

21. Kim J, Kim DW, Kim KI, et al. Compliance of antihypertensive medication and risk of coronavirus disease 2019: a cohort study using big data from the Korean National Health Insurance Service. J Korean Med Sci 2020;35:e232.

22. Bruine de Bruin W, Bennett D. Relationships between initial COVID-19 risk perceptions and protective health behaviors: a national survey. Am J Prev Med 2020;59:157167.

23. Kuba K, Imai $Y$, Rao $S$, et al. A crucial role of angiotensin converting enzyme 2 (ACE2) in SARS coronavirus-induced lung injury. Nat Med 2005;11:875-879.

24. Gu H, Xie Z, Li T, et al. Angiotensin-converting enzyme 2 inhibits lung injury induced by respiratory syncytial virus. Sci Rep 2016;6:19840.

25. Bavishi C, Maddox TM, Messerli FH. Coronavirus disease 2019 (COVID-19) infection and renin angiotensin system blockers. JAMA Cardiol 2020;5:745-747. 


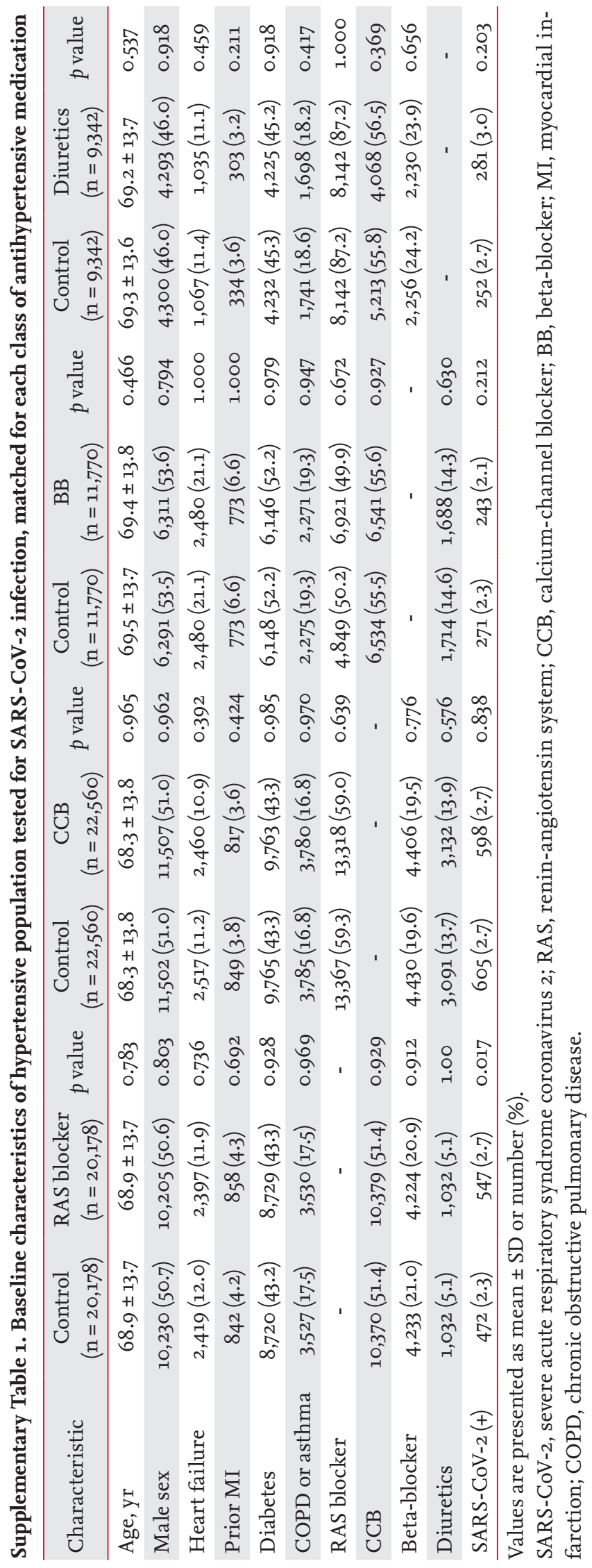




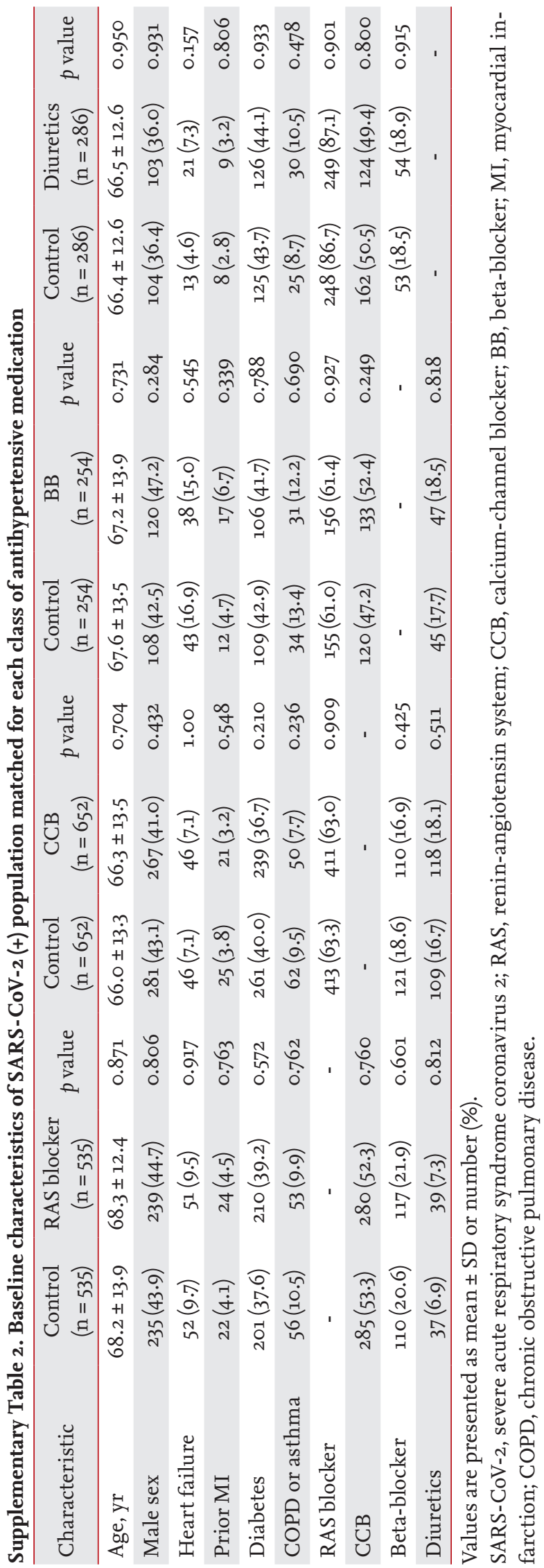


KJIM ${ }^{2}$

Supplementary Table 3. Antihypertensive medication and SARS-CoV-2 susceptibility in propensity score matched patients with hypertension

\begin{tabular}{lcccc}
\hline & Patients on medication & Control & Adjusted OR (95\% CI) & P value \\
\hline RAS blocker & $547 / 20,178(2.7)$ & $472 / 20,178(2.3)$ & $1.16(1.03-1.32)$ & 0.017 \\
Calcium-channel blocker & $598 / 22,560(2.7)$ & $605 / 22,560(2.7)$ & $0.99(0.88-1.11)$ & 0.823 \\
Beta-blocker & $243 / 11,770(2.1)$ & $271 / 11,770(2.3)$ & $0.89(0.75-1.07)$ & 0.212 \\
Diuretics & $281 / 9,342(3.0)$ & $252 / 9,342(2.7)$ & $1.12(0.94-1.33)$ & 0.215 \\
\hline
\end{tabular}

Values are presented as SARS-CoV-2 (+)/total no. (\%). Hypertensive patients on RAS blockers showed higher SARS-CoV-2 positive rate than subjects without RAS blockers after matching for propensity score. Patients were propensity score matched for age, sex, heart failure, diabetes, history of myocardial infarction, asthma or chronic obstructive pulmonary disease, and other classes of medication.

SARS-CoV-2, severe acute respiratory syndrome coronavirus 2; OR, odds ratio; CI, confidence interval; RAS, renin-angiotensin system. 
Supplementary Table 4. Association between each class of antihypertensive medication and clinical outcomes in patients with hypertension

\begin{tabular}{|c|c|c|c|c|c|c|}
\hline \multirow{2}{*}{ Outcome } & \multicolumn{3}{|c|}{$\begin{array}{l}\text { SARS-CoV-2 (+) with HTN } \\
(\mathrm{n}=1,609)\end{array}$} & \multicolumn{3}{|c|}{ Matched population } \\
\hline & No. of events & $\begin{array}{c}\text { Adjusted OR } \\
(95 \% \mathrm{CI})\end{array}$ & $p$ value & No. of events & $\begin{array}{l}\text { Adjusted OR } \\
(95 \% \mathrm{CI})\end{array}$ & $p$ value \\
\hline RAS blocker & & \multicolumn{5}{|c|}{ 1,070 (535 pairs) ${ }^{\mathrm{a}}$} \\
\hline Death & 165 & $0.81(0.56-1.17)$ & 0.265 & 127 & $0.88(0.59-1.33)$ & 0.545 \\
\hline ICU/death & 378 & $0.95(0.73-1.22)$ & 0.669 & 276 & $1.05(0.79-1.40)$ & 0.720 \\
\hline $\mathrm{O}_{2} / \mathrm{ICU} /$ death & 614 & $1.01(0.80-1.27)$ & 0.956 & 439 & $0.99(0.77-1.28)$ & 0.939 \\
\hline Calcium-channel blocker & & \multicolumn{5}{|c|}{$1,304\left(65^{2} \text { pairs }\right)^{\mathrm{a}}$} \\
\hline Death & 165 & $0.87(0.60-1.24)$ & 0.437 & 130 & $1.05(0.61-1.80)$ & 0.875 \\
\hline ICU/death & 378 & $1.01(0.79-1.29)$ & 0.943 & 304 & $1.15(0.88-1.51)$ & 0.305 \\
\hline $\mathrm{O}_{2} / \mathrm{ICU} /$ death & 614 & $1.07(0.86-1.33)$ & 0.556 & 502 & $1.17(0.92-1.48)$ & 0.208 \\
\hline Beta-blocker & & \multicolumn{5}{|c|}{$508(254 \text { pairs })^{\mathrm{a}}$} \\
\hline Death & 165 & $1.21(0.78-1.89)$ & 0.404 & 69 & $1.01(0.57-1.78)$ & 0.976 \\
\hline ICU/death & 378 & $1.18(0.86-1.61)$ & 0.303 & 146 & $0.90(0.60-1.36)$ & 0.626 \\
\hline $\mathrm{O}_{2} / \mathrm{ICU} /$ death & 614 & $1.05(0.79-1.40)$ & 0.744 & 225 & $0.76(0.52-1.10)$ & 0.143 \\
\hline Diuretics & & \multicolumn{5}{|c|}{$572(286 \text { pairs })^{\mathrm{a}}$} \\
\hline Death & 165 & $1.12(0.70-1.80)$ & 0.637 & 56 & $1.21(0.66-2.22)$ & 0.544 \\
\hline ICU/death & 378 & $0.90(0.65-1.25)$ & 0.532 & 128 & $0.85(0.57-1.29)$ & 0.453 \\
\hline $\mathrm{O}_{2} / \mathrm{ICU} /$ death & 614 & $0.86(0.64-1.15)$ & 0.301 & 211 & $0.82(0.57-1.18)$ & 0.293 \\
\hline
\end{tabular}

Patients on each class of medication were matched to patients not using that medication according to propensity scores.

SARS-CoV-2, severe acute respiratory syndrome coronavirus 2; HTN, hypertension; OR, odds ratio; CI, confidence interval; RAS, renin-angiotensin system; ICU, intensive care unit.

${ }^{\mathrm{a}}$ Number of matched population. 